\title{
Determination of Colour-Contrast Sensitivity of the Retina
}

\author{
A. KubARKo ${ }^{a}$, V. Firago ${ }^{b}$ And O. Hotra ${ }^{c, *}$ \\ ${ }^{a}$ Human Physiology Department, Belarusian State Medical University, Minsk, pr. Dzerginsky, 183, 220116, Belarus \\ ${ }^{b}$ Belarusian State University, Department of Quantum Radiophysics and Optoelectronics \\ Minsk, 4, pr. Nezaliezhnasti, 220030, Belarus \\ ${ }^{c}$ Lublin University of Technology, Lublin University of Technology, Faculty of Electrical Engineering \\ and Computer Science, Nadbystrzycka 38a, 20-618 Lublin, Poland
}

\begin{abstract}
The computer methods for determining the map of colour-contrast sensitivity of the retina, suitable for mass screening of the earlier disturbances of visual and circulatory systems of humans, are discussed. The expressions for calculation, and the results of the determination of the threshold values of the photon fluxes at which young people detect the light stimulus in the shape of the red square with the side $2 \mathrm{~mm}$, formed on the screen of CRT display, are given.
\end{abstract}

DOI: 10.12693/APhysPolA.125.1367

PACS: 85.60.-q, 42.79.-e, 42.15.Eq, 07.07.Df, 42.79.Pw

\section{Introduction}

There is a number of psychophysical and electrophysiological methods allowing for determining early changes of visual functions, light, colour and contrast sensitivities caused by pathologies in the vessels, nerve, endocrinal, and other systems.

One of the most informative methods of determination of the preservation of visual functions is the computer static perimetry, at which the visual objects of constant brightness of different levels are shown for observers. The application of stimuli of constant brightness and size allows for estimating the light sensitivity of different regions of the retina. As the parameters of the retina are characterised by high individual variability, the intervals of group norm are set in the perimeters. Such set of the variants of norm decreases the sensitivity of the method, and complicates the earlier revealing of the light sensitivity. In different models of modern static perimeters, the different background brightness of the display, the range and number of the steps of the stimuli brightness change, the algorithms of the threshold determination, and the intervals of norm are employed. This does not contribute to the standardisation of the interpreting and the comparison of the obtained results.

As the result, the elaboration of new methods of light sensitivity determination offering for higher efficiency of the diagnostics of its early changes becomes an actual task [1].

\section{Methods}

While choosing the method of light and colour-contrast sensitivities of the central region of visual field, the modi-

\footnotetext{
*corresponding author; e-mail: o.hotra@pollub.pl
}

fied method of topography (topography mapping) of contrast and colour sensitivity investigation was the basic method [2].

It is known that for every point of visual field there is a certain minimum level (threshold) of contrast- and colour sensitivity, dependent on displacement density of cones sensitive to contrast and some colour. In order to reveal it, in our investigations the light stimuli of red colour in the shape of a square (of the side $a$ equal to $2 \mathrm{~mm}$ ) were given for observer on display in series. The brightness of stimulus luminescence during $5.6 \mathrm{~s}$ was increased from the background value up to the maximum value. For light sensitivity mapping, the stimuli placed eccentrically in the visual field of observer within the limit of $20^{\circ}$ from the central fovea of the retina were employed. In all, 141 points were used.

During the investigation process, the observer looks with the tested eye on weakly luminescent cross, placed in the display centre. The other eye was hidden using an opaque screen. The distance between the display and the eye was $30 \mathrm{~cm}$. The head of the observer was fixed on special support, adjusted vertically in order to place the visual axis of the eye at the level of display centre. The coordinates and time of putting of light stimuli were determined using special algorithm [3] based on pseudo-probable law in order to eliminate the process of adaptation to the sequence of stimuli appearing in the display.

The observer ought to press the button on the computer keyboard at the moment of noticing the appearance of the luminous point on the display, without shifting the eyesight on it. After pressing the button, the computer marked on the creating map of colour-contrast sensitivity the obtained value of the signal which controls the stimuli brightness, and initiated the process of delivering the next stimulus. In the case of the absence of observer's reaction to the stimulus - for example if the image is in the region of blind spot - the algorithm repeats the delivery of stimulus with the same coordinates. 
As the light sensitivity of human eye is high, the Samsung SyncMaster 550b display, based on CRT with display size $32 \times 24 \mathrm{~cm}^{2}$, was employed. On the contrary to other types of displays, the displays based on CRT exhibit virtually no luminescence of the display.

After finishing the investigation, the map of the threshold light or colour-contrast sensitivity of the investigated eye was created on the display. On the map, the fixed values of stimulus brightness in arbitrary units were marked with numbers in the points described with certain coordinates.

For the sake of standardisation of the investigation procedure and calculation of the values of the light flux falling on the retina, both the calibration of stimuli luminescence brightness in absolute physical quantities, and the equipment for checking the size of pupil of investigated eye are required.

\section{Determination of the absolute brightness of the stimuli luminescence}

In order to determine the brightness of the luminescence of the formed stimuli depending on the controlled digital signal $d_{\mathrm{sc}}$ put on the controller of the display, the photometer comprising the digital monochromatic video camera mvBlueFOX $223 \mathrm{G}$ and a notebook, was employed. The video camera was placed $z=30 \mathrm{~cm}$ from the display, in such position that the axis of its visual field was perpendicular to the display plane, and passed through the display centre.

The spectral flux, put on the matrix light sensitive element of the area $s_{\mathrm{el}}$, which was fully covered by stimulus image, can be expressed as follows:

$$
\Phi_{\mathrm{el}}(\lambda)=\tau_{\mathrm{ob}} s_{\mathrm{el}} M(\lambda) \frac{d^{2}}{4 f^{2}}\left(1-\frac{f}{z}\right)^{2}=K M(\lambda),
$$

where $f, d$ and $\tau_{\mathrm{ob}}$ are the focus distance, the diameter, and the transmission coefficient of the lens, respectively, $s_{\mathrm{el}}$ is the area of the matrix element, $M(\lambda)$ is the spectral luminosity of the stimulus, and $K$ is the coefficient which accounts for the parameters of the lens and the measuring circuit. At $a / z<0.1$, the coefficient $K$ is approximately equal to

$$
K \approx \tau_{\mathrm{ob}} \frac{d^{2}}{4 f^{2}}\left(1-\frac{f}{z}\right)^{2} .
$$

Introducing the absolute spectral current sensitivity of the matrix elements

$$
S(\lambda)=S_{\max } s(\lambda),
$$

where $S_{\max }$ is the maximum absolute sensitivity, $s(\lambda)$ is the relative spectral sensitivity, and integrating the expression (3) over $\lambda$, one can obtain the photocurrent formed in one element of the matrix

$$
\begin{aligned}
I_{\mathrm{ph}} & =S_{\max } \int_{\lambda_{1}}^{\lambda_{2}} s(\lambda) \Phi_{\mathrm{el}}(\lambda) \mathrm{d} \lambda \\
& =K s_{\mathrm{el}} S_{\max } \int_{\lambda_{1}}^{\lambda_{2}} s(\lambda) M(\lambda) \mathrm{d} \lambda .
\end{aligned}
$$

The charge $Q$ accumulated during exposure time $\tau$ at the constant value of the display luminosity $M(\lambda)$ in the light sensing elements of the matrix will be proportional to the photocurrent $I_{\mathrm{ph}}: Q=I_{\mathrm{ph}} \tau$. The digital signal $D$ formed by the controller of the video camera is dependent on the coefficient of transformation of the charge into the digital code $k_{\mathrm{QD}}$, i.e. $D=k_{\mathrm{QD}} Q=k_{\mathrm{QD}} I_{\mathrm{ph}} \tau$.

As the exposure time $\tau$ depends on the illumination of the image, for determining the luminescence brightness of the display it is better to use the speed of digital signal increase $V$ :

$$
\begin{aligned}
V & =\frac{D}{\tau}=\frac{k_{\mathrm{QD}} Q}{\tau}=k_{\mathrm{QD}} I_{\mathrm{ph}} \\
& =k_{\mathrm{QD}} K s_{\mathrm{el}} S_{\max } \int_{\lambda_{1}}^{\lambda_{2}} s(\lambda) M(\lambda) \mathrm{d} \lambda,
\end{aligned}
$$

which is proportional to the photocurrent.

The speed $V$ depends on a few parameters, and spectral characteristics of the matrix and source of luminescence which could be set. This allows for $V$ calculation. The unknown values $k_{\mathrm{QD}}$ and $S_{\max }$ in the last expression are not given in the documentation of the matrix and camera. As they are constants, one can unite them in one unknown value $k_{\mathrm{QDS}}$ which should be determined at calibration stage of the photometer. By calibration, the correction coefficient $k_{\text {cor }}$ can be found. Multiplying $k_{\text {cor }}$ by the calculated value $k_{\mathrm{QDS}}$, the coincidence of the calculated $V_{\text {cal }}$ and experimental $V_{\text {ex }}$ values can be achieved.

The speed of digital signal increase at calibration $V_{\text {ex }}$ can be determined, if the standard emitter is put on display place. As the brightness of standard emitters in the visual part of the spectrum is usually high, in order to decrease the value of registered fluxes it is appropriate to employ the optical filter with transmission $\tau_{\mathrm{f}}(\lambda)$ in the measurement circuit. If the standard emitter is the model of the absolutely black body (ABB), then the following integral expression for calculating the speed of digital signals $V_{\text {cal }}$ should be used:

$$
\begin{aligned}
V_{\text {cal }} & =k_{\mathrm{QDS}} K s_{\mathrm{el}} \int_{\lambda_{1}}^{\lambda_{2}} \tau_{\mathrm{f}}(\lambda) s(\lambda) M_{\mathrm{BB}}(\lambda, T) \mathrm{d} \lambda \\
& =k_{\mathrm{QDS}} K s_{\mathrm{el}} c_{1} \int_{\lambda_{1}}^{\lambda_{2}} \tau_{\mathrm{f}}(\lambda) s(\lambda) \frac{1}{\lambda^{5}} \\
& \times\left(\mathrm{e}^{c_{2} /(\lambda T)}-1\right)^{-1} \mathrm{~d} \lambda,
\end{aligned}
$$

where $M_{\mathrm{BB}}(\lambda, T)=\frac{c_{1}}{\lambda^{5}}\left(\mathrm{e}^{c_{2} /(\lambda T)}-1\right)^{-1}$ is the surface luminosity of the ABB model, $c_{1}$ and $c_{2}$ are the first and the second constants of the luminescence radiation.

Measurement of the experimental value of the speed $V_{\text {ex }}$ during calibration allows for determining the value of the correction coefficient $k_{\text {cor }}=V_{\text {ex }} / V_{\text {cal }}$.

At investigation of the contrast-colour sensitivity, the increasing brightness of the red stimulus was applied. The experimental dependence of brightness (the points in Fig. 1) on the value of the controlled digital signal, at the background luminescence of the display (residual itself, and reflected), of the approximate brightness $L_{\mathrm{f}}=0.8 \mathrm{~mW} /\left(\mathrm{m}^{2} \mathrm{sr}\right)$ is plotted in Fig. 1. 


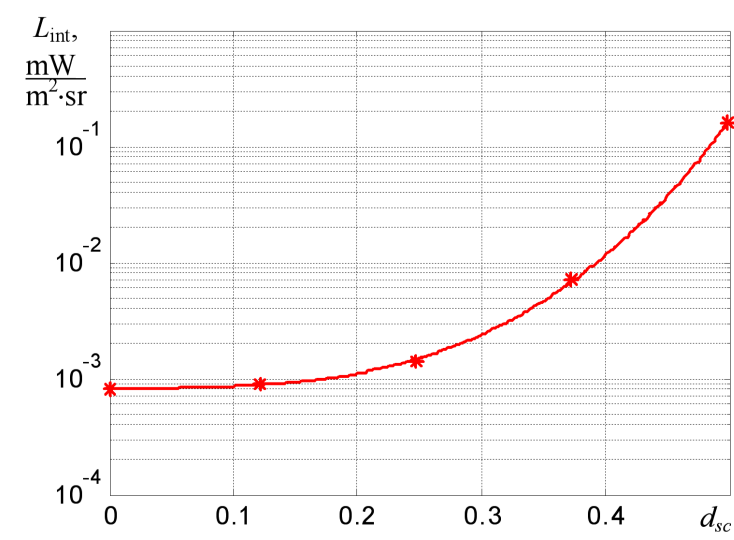

Fig. 1. Relationship between the integral brightness of the luminescence of $R$ elements of the display Samsung Master $550 \mathrm{~b}$ and the value of the controlled digital signal at $78 \%$ brightness and $100 \%$ contrast.

\section{Calculation of the illumination of the retina}

Knowing the sizes of the formed stimulus and the dependence of its brightness on the value of the controlled signal, the illumination of the retina can be simply calculated. This allows for estimation of the threshold flux of the photons per one photoreceptor in average.

Using the expression for thin lens, one can obtain the expression for integral illumination of the stimulus image on the eye retina

$$
E_{\mathrm{ims}} \approx \tau_{\text {eye }} \pi L_{\text {int }} \frac{D_{\text {eye }}^{2}}{4 f_{\text {eye }}^{2}}\left(1-\frac{f_{\text {eye }}}{z}\right)^{2},
$$

where $\tau_{\text {eye }}$ is the coefficient of transmittance of the ocular medium, which is in the range from 0.5 to $0.9, D_{\text {eye }}$ is the diameter of the pupil, $f_{\text {eye }}$ is the focus distance at focusing of the eyesight on the cross in the centre of the display placed on distance $30 \mathrm{~cm}$ from the eye. The average value of $f_{\text {eye }}$ equals $2 \mathrm{~cm}$. The diameter of the pupil at dark adaptation equals $6 \mathrm{~mm} \pm 1 \mathrm{~mm}$. The length of the side of the stimulus image on the retina is determined from the following expression: $b=a f_{\text {eye }} /\left(z-f_{\text {eye }}\right)$. At $a=2 \mathrm{~mm}$ the value $b$ is approximately equal to $143 \mu \mathrm{m}$. The square of the stimulus image approximately equals: $S_{\mathrm{ims}}=b^{2} \approx 2 \times 10^{-8} \mathrm{~m}^{2}$.

The dependence of the illumination of the retina $E_{\mathrm{im}}$ (in the region of the red stimulus image) on the value of the controlled digital signal at different values of $\tau_{\text {eye }}$ taking into account the background luminescence of the display $L_{\mathrm{f}}$, is shown in Fig. 2. The radiation flux of the stimulus falling on the retina at known dependence $E_{\text {ims }}\left(d_{\mathrm{sc}}\right)$, and the square of the image $S_{\mathrm{ims}}$, can be easily calculated

$$
\Phi_{\text {ims }}=E_{\text {ims }}\left(d_{\text {sc }}\right) S_{\text {ims }} .
$$

It can be seen in Fig. 2 that the illumination of the retina in the region of the image of a given light stimulus is determined by the total intensity of two light fluxes: constant background flux, and local additional flux de-

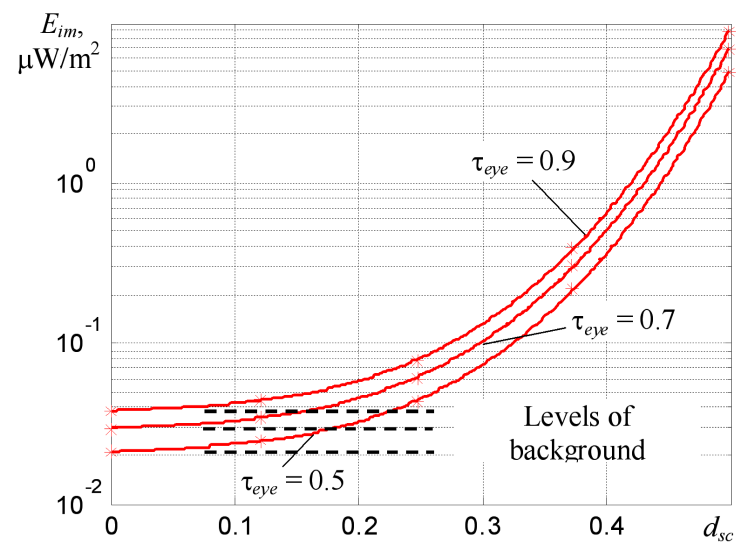

Fig. 2. Relationship between the integral illumination of the red stimulus image on the retina and the value of the controlled digital signal.

termined by stimulus brightness. As mainly the cones take part in the forming of the visual image (the photopic vision is used), the threshold values of the illumination should be much higher than at investigation of light (scotopic) threshold sensitivity. At full dark adaptation, the light perception formed by receptor field of rods, appears at falling of approximately from 30 to 70 photons on cornea during several tens of ms. The sensitivity of cones is near 170-600 photons [4]. Therefore, it is accepted that the average absolute sensitivity of the photopic vision is at least 5 times worse than periphery scotopic ones, i.e. it should be a few thousands of photons in average.

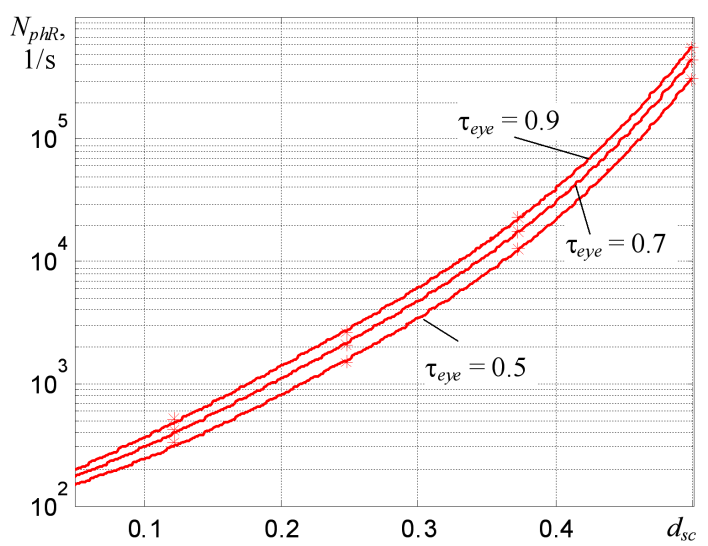

Fig. 3. Relationship between the value of the average photon flux $N_{\text {phr }}$ falling on the retina per $1 \mathrm{~s}$ and the value of the controlled digital signal.

Subtracting of the background luminescence, the relationship between the average photon flux falling on the retina in the region of the stimulus image, and the value of the controlled signal can be obtained. Assuming that the mean value of the luminescence wavelength of the red luminophor equals $\lambda_{\mathrm{R}}=630 \mathrm{~nm}$, the energy of one photon can be found: $E_{\mathrm{R}}=h c / \lambda_{\mathrm{R}}$, and the relationship 
between $N_{\mathrm{phR}}$ and $d_{\mathrm{sc}}$ can be plotted (Fig. 3 ):

$$
N_{\mathrm{phR}}=\frac{\Phi_{\mathrm{ims}}\left(d_{\mathrm{sc}}\right)-\Phi_{\mathrm{f}}}{E_{\mathrm{phR}}}=\frac{\left[E_{\mathrm{ims}}\left(d_{\mathrm{sc}}\right)-E_{\mathrm{ims}}(0)\right] S_{\mathrm{ims}}}{h c / \lambda_{\mathrm{R}}} .
$$

The measurements carried out, and calculations based on the described methods show that the colour-contrast sensitivity of the visual system of the healthy young people of the age of $18-30$, for red colour radiation is equal in average just little more than 1000 photons/s (Fig. 3), and worsens with age. The reaction on stimulus of young people of the same age with increased arterial blood pressure (arterial hypertension of 1-2 degrees) requires $16 \%$ higher flux of photons.

\section{Summary}

The proposed computer methods for determining the map of colour-contrast sensitivity of the retina are easy in practical implementation, show good repeatability, and can form the basis for elaborating the equipment for mass screening detection of the early disturbances of the functions of the visual and vascular systems.

\section{References}

[1] A. Kubarko, O. Hotra, N. Kubarko, D. Alexandrov, Ju. Kubarko, I. Goursky, Przeglad Elektrotechniczny 89, 309 (2013).

[2] N. Accornero, S. Rinalduzzi, M. Capozza, E. Millefiorini, G.C. Filligoi, L. Capitanio, Mult. Scler. 4, 79 (1998).

[3] A. Kubarko, B. Chuprin, N. Kubarko, J. Kubarko, Teorija i Praktika Medicyny 3, 195 (2002) (in Russian).

[4] D. Koenig, H. Hofer, J. Vision 11(1), 21 (2011). 